\title{
Design and Implementation of a Fault-Tolerant Magnetic Bearing System for MSCMG
}

\author{
Enqiong Tang ${ }^{1,2}$ and Bangcheng Han ${ }^{1,2}$ \\ ${ }^{1}$ School of Instrument Science and Optoelectronics Engineering, Beihang University, Beijing 100191, China \\ ${ }^{2}$ Science and Technology on Inertial Laboratory, Beijing 100191, China \\ Correspondence should be addressed to Bangcheng Han; hanbangcheng@buaa.edu.cn
}

Received 2 October 2013; Accepted 7 November 2013

Academic Editor: Sebastian Anita

Copyright (C) 2013 E. Tang and B. Han. This is an open access article distributed under the Creative Commons Attribution License, which permits unrestricted use, distribution, and reproduction in any medium, provided the original work is properly cited.

\begin{abstract}
The magnetically suspended control moment gyros (MSCMGs) are complex system with multivariable, nonlinear, and strongly gyroscopic coupling. Therefore, its reliability is a key factor to determine whether it can be widely used in spacecraft. Fault-tolerant magnetic bearing systems have been proposed so that the system can operate normally in spite of some faults in the system. However, the conventional magnetic bearing and fault-tolerant control strategies are not suitable for the MSCMGs because of the movinggimbal effects and requirement of the maximum load capacity after failure. A novel fault-tolerant magnetic bearing system which has low power loss and good robust performances to reject the moving-gimbal effects is presented in this paper. Moreover, its maximum load capacity is unchanged before and after failure. In addition, the compensation filters are designed to improve the bandwidth of the amplifiers so that the nutation stability of the high-speed rotor cannot be affected by the increasing of the coil currents. The experimental results show the effectiveness and superiority of the proposed fault-tolerant system.
\end{abstract}

\section{Introduction}

Control moment gyros are capable of producing significant torques and handling large quantities of momentum over long periods of time. Consequently, they are commonly employed to reorient large spacecraft structures by using large torque capacity [1]. A control moment gyro with magnetic bearings that comprises of a rapidly spinning rotor mounted on a gimbal is accordingly called a Single-Gimbal Magnetically Suspended control moment gyro (SGMSCMG). Magnetic bearing are ideally suited for high-speed and vacuum applications due to their contact free operation, low friction losses, adjustable damping, controllable vibration [2], and stiffness characteristics and the fact that no lubricants are necessary.

However, the most significant drawback of magnetic bearing technology is that even a single failure within one subassembly (digital controller, magnetic actuator, amplifier, and position sensors) may lead to the complete system failure. Fault-tolerant magnetic bearing systems have been proposed so that the system can operate in spite of some faults in the system.
The strong coupling property and redefined remaining coil currents make the heteropolar magnetic bearing possible to produce desired force resultants in the $x$ - and $y$-directions even when some coils fail. A bias current linearization method to accommodate the fault tolerance of magnetic bearings was developed, so the redistribution matrix that linearizes control forces can be obtained even if one or more coils fail $[3,4]$. Fault-tolerant control of heteropolar magnetic bearings has been demonstrated on a five-axis, flexible rotor test rig with three CPU failures and two (out of eight) adjacent coil failures [5]. Current distribution matrix for heteropolar magnetic bearings was extended to cover five pole failures out of eight poles $[6,7]$ and for the case of significant effects of material path reluctance and fringing [8].

The fault-tolerant approaches outlined above utilize the current distribution matrix that changes the current in each pole after failure in order to achieve linearized and decoupled relations between control forces and control voltages. But calculating the current distribution matrix is typically performed at the magnetic center so that the modeling of the bearing force can be simplified. Therefore, this method is mainly applied to the target whose rotor is always suspended in the 


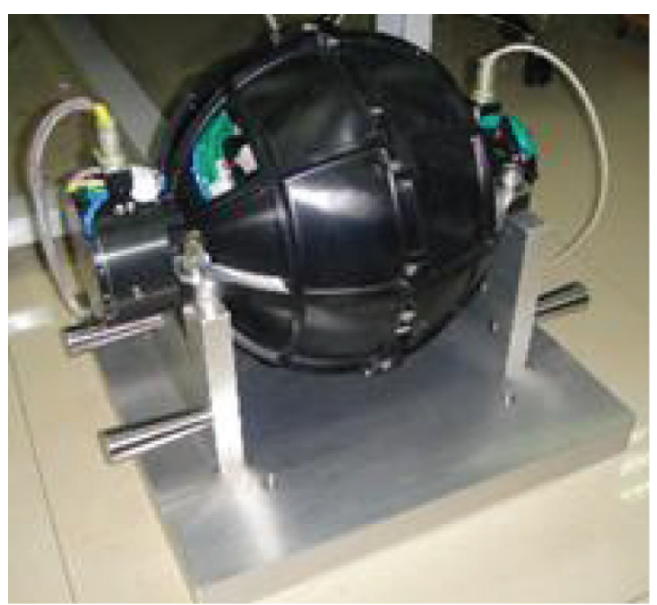

(a)

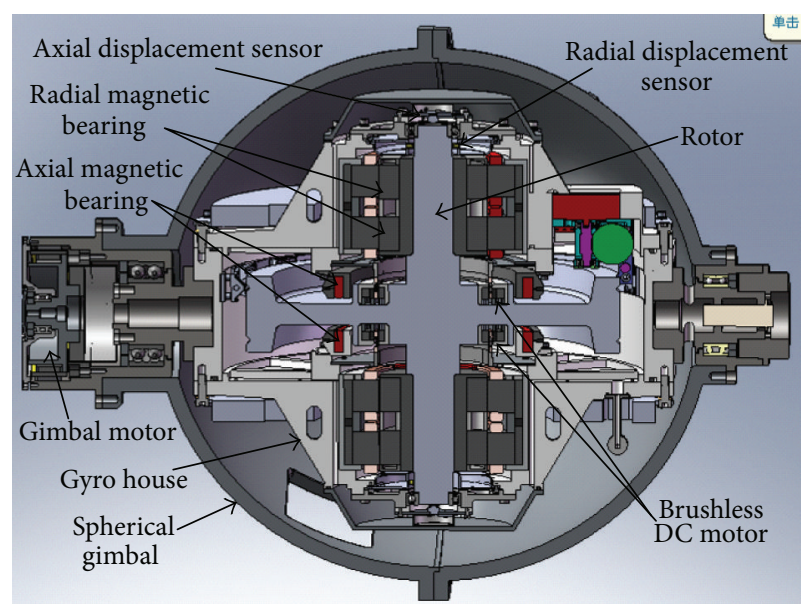

(b)

FIGURE 1: The SGMSCMG and its schematic diagram. (a) Photograph of the SGMSCMG. (b) Schematic diagram of the SGMSCMG.

center, such as Turbo-Molecular Vacuum Pump [9], Flywheel [10], and Magnetically Suspended Motor [11].

The SGMSCMG is a complex system with multivariable, nonlinear, and strongly gyroscopic coupling. Especially the gyroscopic effects [10], moving-gimbal effects [12, 13], and unbalance vibrations make the high-precision control more difficult. The gimbal movement disturbs the magnetically suspended rotor, meanwhile the gyroscopic effects make the movement of the disturbed rotor more complex, which aggravates the runout of the rotor and even endangers the system stability. The traditional control method like cross-feedback [10] plus rate feedforward control [12], inverse system method [13], $\mu$-synthesis control [14], and so forth are used to suppress the gyroscopic effects and moving-gimbal effects.

Although these methods have a certain effect to suppress the moving-gimbal effects, the rotor displacement will still be sharp fluctuations at the moment when the gimbal start rotating. Then the bearing force which has been decoupled and linearized by the current distribution matrix will present strongly coupling and nonlinear characteristic again when the rotor is away from the magnetic center. Therefore, for the fault-tolerant control of the SGMSCMG, the application of the current distribution matrix will be hindered.

In addition, the maximum load capacity of the magnetic bearing with general structure will decrease after failure, and it is restricted if we improve the load capacity by only increasing the coil current owing to the magnetic saturation characteristics of the permanent magnet-biased radial magnetic bearing (PMRMB). The load capacity of the magnetic bearing is in proportion to the torque provided by the SGMSCMG.

The rated torque is a very important performance index of the SGMSCMG. If the SGMSCMG cannot output the rated torque when the fault-tolerant control method is used after failure, these methods are incomplete and also cannot be accepted.

In this paper, we designed a fault-tolerant magnetic bearing system for the SGMSCMG. The system can cope with the actuator/amplifier faults even though the rotor is not at the magnetic center. Furthermore, the maximum load capacity of the magnetic bearing will not change in spite of the actuator/amplifier faults.

This paper is organized as follows. Section 2 describes the structure of the novel four-pole PMRMB and the dynamic model of the high-speed shaft. Section 3 analyzes the linear and decoupling characteristics of the four-pole PMRMB. Its load capacity is also presented in this section. Section 4 designs the fault-tolerant controller and the amplifier compensation filters for the SGMSCMG. Section 5 provides the experimental results based on fault-tolerant control operation. Section 6 summarizes the advantages over the four-pole permanent magnet-biased PMRMB.

\section{System Description}

Figure 1 shows the actual appearance and the schematic diagram of the SGMSCMG which is used to control large spacecrafts. The rotor is driven by a brushless DC motor, and supported by the combination of two radial magnetic bearings and two axial magnetic bearings. The displacement of the rotor is detected by four sets of eddy-current sensors. For startups and shutdowns, the rotor has two backup ball bearings which have the radial clearance of $0.1 \mathrm{~mm}$. In the nominal working conditions, the rotational speed of rotor is up to $20000 \mathrm{rpm}$ and the angular velocity of gimbal is $10^{\circ} / \mathrm{s}$.

Figures 2 and 3 show the difference of two kinds of stators of the magnetic bearings. Figure 2 presents an eight-pole PMRMB stator and its lamination in common use [15]. Figure 3 demonstrates the four-pole PMRMB stator and its lamination. The main structure difference between the novel and the conventional four-pole PMRMB is that the controlflux as well as electromagnetic forces of the $x$ - and $y$-dimension distributes in two parallel planes exclusively that is, there are only two poles with coils belonging to one dimension on each core layer, illustrated as Figure 3, where bias-flux path and control-flux path are also shown in Figure 4.

Figure 5 depicts the high-speed rotor of the SGMSCMG. The middle of the rotor is circular and the moment of inertia 


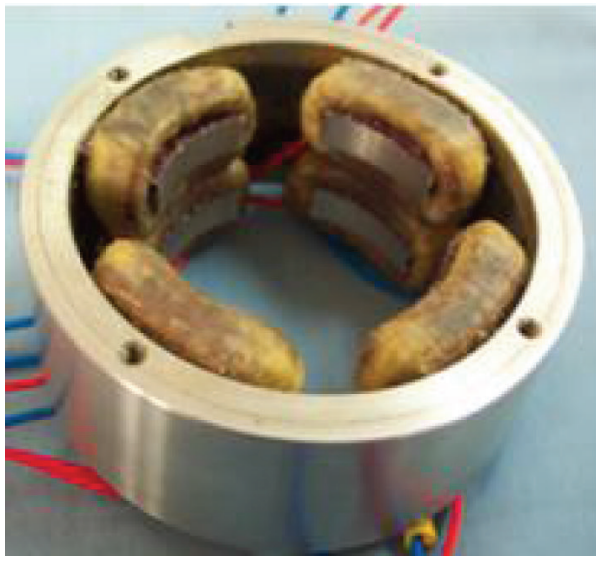

(a)

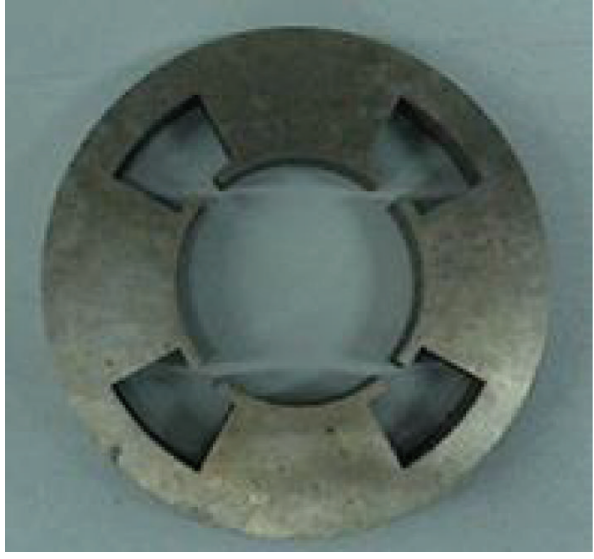

(b)

FIGURE 2: One type of eight-pole PMRMB stator and its lamination. (a) Eight-pole PMRMB stators. (b) Eight-pole stator lamination.

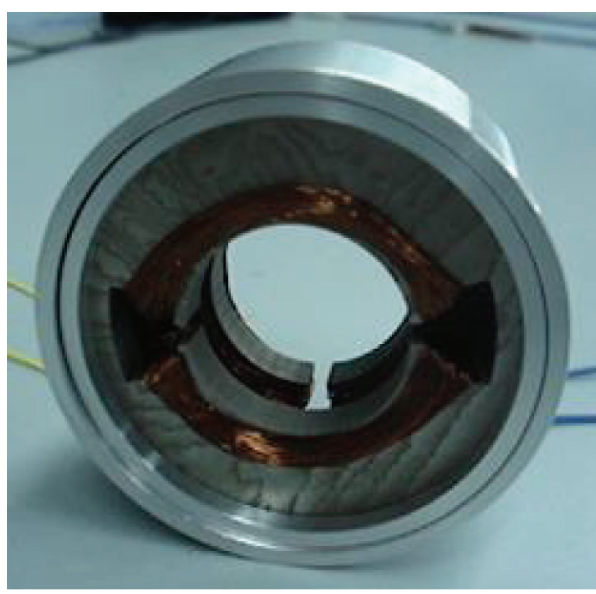

(a)

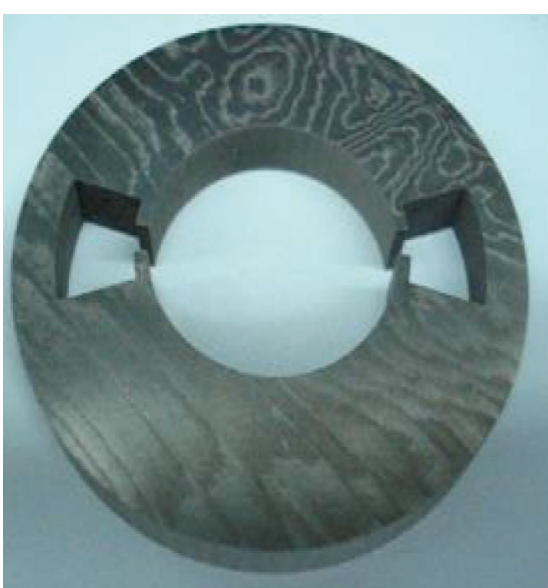

(b)

FIgURE 3: One type of four-pole PMRMB stator and its lamination. (a) Four-pole PMRMB stators. (b) Four-pole stator lamination.

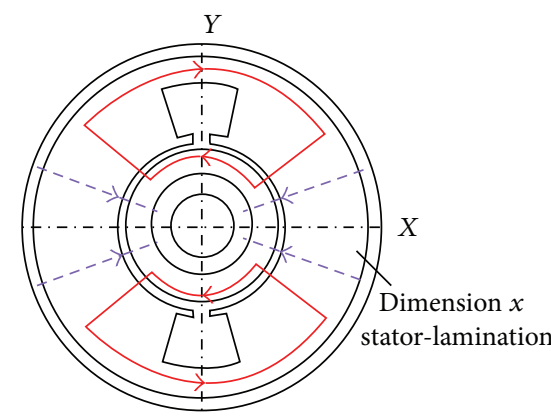

$-\rightarrow-$ Permament magnet flux $\rightarrow-$ Control flux

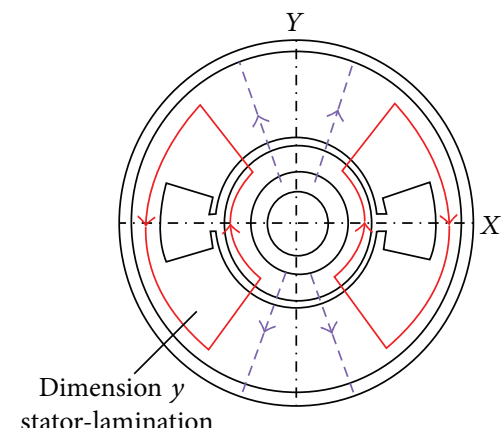

$-\rightarrow-$ - Permament magnet flux

$\rightarrow-$ Control flux

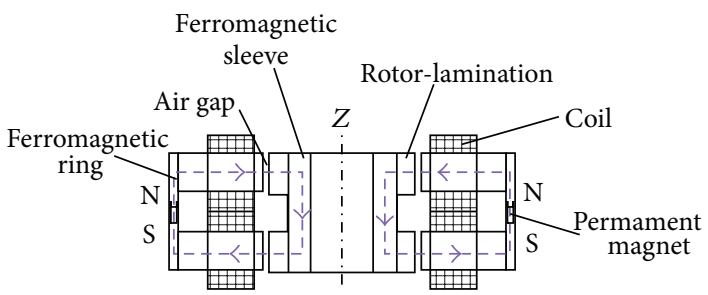

FIGURE 4: Flux path of the four-pole magnetic bearings. 

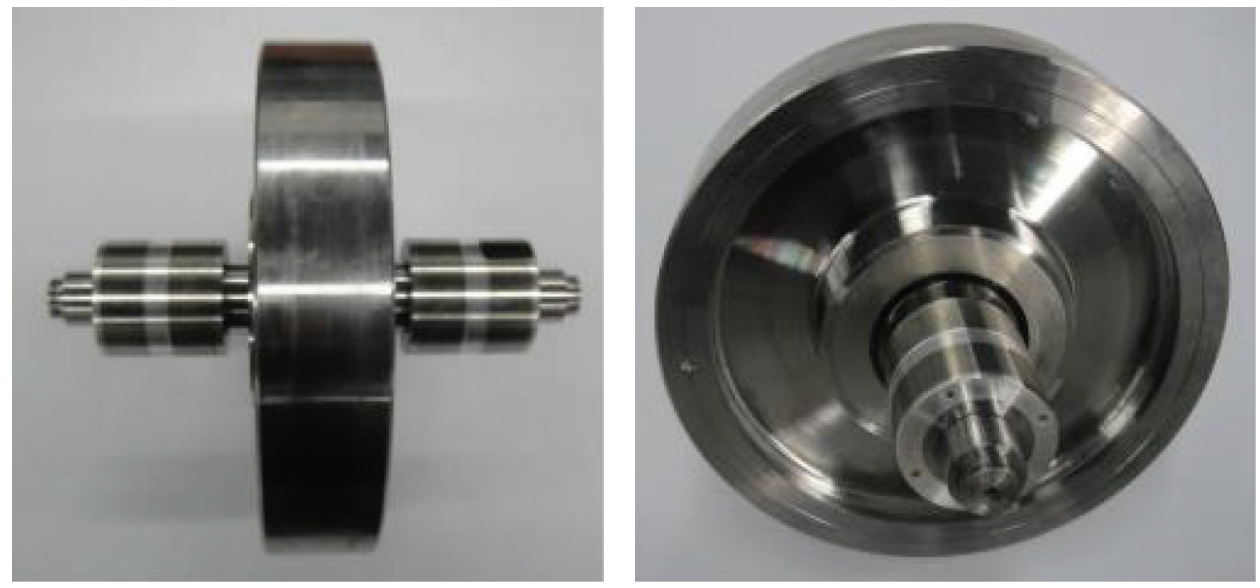

FIGURE 5: High-speed rotor of the SGMSCMG.

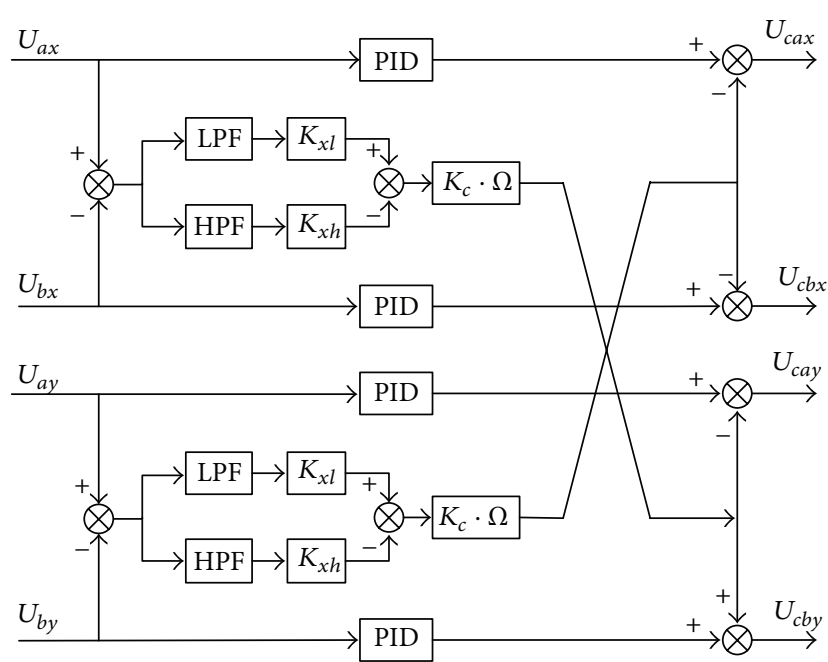

FIGURE 6: Schematic diagram of the cross-feedback control.

is big so that the SGMSCMG can output large torque. Decentralized proportional-integral-derivative (PID) and crossfeedback control system are presently the standard in magnetic bearings [16]. Figure 6 shows the schematic diagram of the cross-feedback control, where LPF and HPF are low-pass filter and high-pass filter, $K_{x l}$ and $K_{x h}$ are the gains of the LPF and HPF, $K_{c}$ is the cross-feedback coefficient, and $\Omega$ is the rotational speed of the rotor.

\section{Modeling and Analysis of the Four-Pole PMRMB}

3.1. Modeling of the Four-Pole PMRMB. Figure 7 shows the equivalent magnetic circuit of the four-pole magnetic bearing. The flux/current relations for this circuit which are obtained by applying Kirchhoff's theory are

$$
\left[\begin{array}{cccc}
R_{x 1} & -R_{x 2} & 0 & 0 \\
R_{\mathrm{pm}} & R_{\mathrm{pm}}+R_{x 2} & R_{y 1} & 0 \\
0 & 0 & R_{y 1} & -R_{y 2} \\
1 & 1 & -1 & -1
\end{array}\right]\left[\begin{array}{c}
\phi_{x 1} \\
\phi_{x 2} \\
\phi_{y 1} \\
\phi_{y 2}
\end{array}\right]
$$

$$
=\left[\begin{array}{cccc}
N_{x 1} & -N_{x 2} & 0 & 0 \\
0 & N_{x 2} & N_{y 1} & 0 \\
0 & 0 & N_{y 1} & -N_{y 2} \\
0 & 0 & 0 & 0
\end{array}\right]\left[\begin{array}{c}
i_{x 1} \\
i_{x 2} \\
i_{y 1} \\
i_{y 2}
\end{array}\right]+\left[\begin{array}{c}
0 \\
H_{\mathrm{pm}} L_{\mathrm{pm}} \\
0 \\
0
\end{array}\right],
$$

where

$$
\begin{array}{ll}
R_{i}=\frac{g_{i}}{\mu_{0} a_{i}} \quad\left(i=x_{1}, x_{2}, y_{1}, y_{2}\right), & g_{x 1}=g_{0}-h_{x}, \\
g_{x 2}=g_{0}+h_{x}, \quad g_{y 1}=g_{0}-h_{y}, & g_{y 1}=g_{0}+h_{y},
\end{array}
$$

where $h_{x}$ and $h_{y}$ are the displacements of the rotor in the $x$ and $y$-direction, $a_{i}$ is the face area of the $i$ th pole of radial MB, and $g_{i}$ is the air gap of the $i$ th pole of radial MB. $g_{0}$ is the air gap of dead pole of HRB. $R_{\mathrm{pm}}$ is the reluctance of permanent magnet, $N$ is the number of turns on the coil, $H_{\mathrm{pm}}$ is the coercive force of permanent magnet, and $L_{\mathrm{pm}}$ is the length of permanent magnet.

Then (1) can be described as

$$
R \Phi=N I+H
$$

Let

$$
I_{c}=\left[\begin{array}{ll}
i_{c x} & i_{c y}
\end{array}\right]^{T}
$$

represent the control currents provided by amplifiers. The currents distributed to the coil are related to the control current vector with the matrix $T_{c}$. Fault conditions are represented using the matrix $K$ that has a null row for each faulted pole. Then the coil currents become

$$
I=K T_{c} I_{c}
$$

where

$$
K=\operatorname{diag}\left(\begin{array}{llll}
k_{x 1} & k_{x 2} & k_{y 1} & k_{y 2}
\end{array}\right), \quad T_{c}=\left[\begin{array}{cc}
1 & 0 \\
-1 & 0 \\
0 & 1 \\
0 & -1
\end{array}\right],
$$




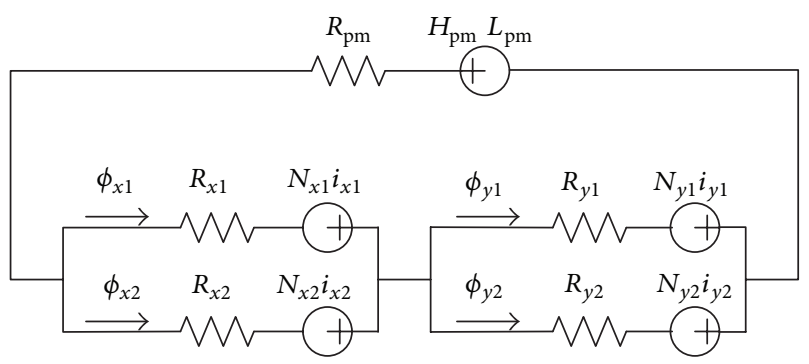

FIgURE 7: Equivalent magnetic circuit of the four-pole PMRMB.

where $k_{x 1}, k_{x 2}, k_{y 1}$, and $k_{y 2}$ are fault flag of the corresponding faulted pole. For example, if coil X1 fail, $K=\operatorname{diag}\left(\begin{array}{llll}0 & 1 & 1 & 1\end{array}\right)$.

The magnetic flux vector is then described as

$$
\Phi=R^{-1}\left(N K T I_{c}+H\right)
$$

Let $A$ represent a diagonal matrix of pole gap areas; then

$$
A B=\Phi
$$

The flux density vector can be solved by (7)-(8)

$$
B=A^{-1} \Phi=A^{-1} R^{-1}\left(N K T I_{c}+H\right)=V I_{c}+B_{\text {bias }},
$$

where

$$
V=A^{-1} R^{-1} N K T, \quad B_{\text {bias }}=A^{-1} R^{-1} H .
$$

Equation (9) shows that the control flux varies with the control currents and the rotor displacements, but the bias flux varies only with the rotor displacements. The magnetic forces along the direction determined from the Maxwell stress tensor are given as

$$
\begin{aligned}
& f_{x}=B^{T} D_{x} B, \\
& f_{y}=B^{T} D_{y} B,
\end{aligned}
$$

where

$$
\begin{aligned}
& D_{x}=\operatorname{diag}\left[\begin{array}{llll}
\frac{A}{2 \mu_{0}} & -\frac{A}{2 \mu_{0}} & 0 & 0
\end{array}\right], \\
& D_{y}=\operatorname{diag}\left[\begin{array}{llll}
0 & 0 & \frac{A}{2 \mu_{0}} & -\frac{A}{2 \mu_{0}}
\end{array}\right] .
\end{aligned}
$$

\begin{tabular}{|c|c|}
\hline No. & $K$ \\
\hline 1 & $\operatorname{diag}\left(\left[\begin{array}{llll}0 & 1 & 1 & 1\end{array}\right]\right)$ \\
\hline 2 & $\operatorname{diag}\left(\left[\begin{array}{llll}0 & 1 & 0 & 1\end{array}\right]\right)$ \\
\hline
\end{tabular}

The magnetic force along the direction are described as

$$
\begin{aligned}
& f_{x}=I_{c}^{T} M_{1} I_{c}+M_{2} I_{c}+B_{\text {bias }}{ }^{T} D_{x} B_{\text {bias }} \\
& f_{y}=I_{c}^{T} M_{3} I_{c}+M_{4} I_{c}+B_{\text {bias }}{ }^{T} D_{y} B_{\text {bias }}
\end{aligned}
$$

where

$$
\begin{array}{ll}
M_{1}=V^{T} D_{x} V, & M_{2}=2 B_{\text {bias }}{ }^{T} D_{x} V, \\
M_{3}=V^{T} D_{y} V, & M_{4}=2 B_{\text {bias }}{ }^{T} D_{y} V .
\end{array}
$$

TABLe 1: Failure Mode.

TABLE 2: Bearing specifications.

\begin{tabular}{lc}
\hline Parameter & Value \\
\hline$l_{m}(\mathrm{~m})$ & 0.0435 \\
$H_{\mathrm{pm}}$ & 796000 \\
$L_{\mathrm{pm}}(\mathrm{mm})$ & 5 \\
$A\left(\mathrm{~mm}^{2}\right)$ & 380 \\
$B_{s}(\mathrm{~T})$ & 0.8 \\
$N$ & 95 \\
$g_{0}(\mu \mathrm{m})$ & 200 \\
\hline
\end{tabular}

The coupling and nonlinear magnetic forces can also be linearized at the bearing center position and the zero coil current when the MB system has no fault by using Taylor series expansion

$$
\begin{aligned}
& f_{x}=k_{i} i_{c x}+k_{h} h_{x}, \\
& f_{y}=k_{i} i_{c y}+k_{h} h_{y} .
\end{aligned}
$$

3.2. Analysis of the Decoupling Characteristic. In all, there are eight different ways that a bearing can fail among the four coils. All of these failures are, however, described by only 2 unique failure maps due to the symmetry of the bearing; all other mappings are simply rotations and permutations of these unique maps. If a " 1 " represents an active coil and a " 0 " represents an inactive coil, then the unique configurations can be described as Table 1. The specifications of the bearings are summarized in Table 2, where $B_{s}$ is the saturation flux density.

Obviously, the coil failure mainly affects the first two items at the right of (13). According to the parameters in Table 2, when the magnetic bearing system is faultless, we can obtain the following results:

$$
\begin{aligned}
& M_{1}=M_{2}=0_{2 \times 2}, \\
& M_{3}=\left[\begin{array}{ll}
160.38 & 0
\end{array}\right], \\
& M_{4}=\left[\begin{array}{ll}
0 & 160.38
\end{array}\right] .
\end{aligned}
$$

Then, the magnetic force can be described as

$$
\begin{aligned}
& f_{x}=160.38 i_{c x}-0.57 h_{x}, \\
& f_{y}=160.38 i_{c y}-0.57 h_{y} .
\end{aligned}
$$

Now analyze the changes in character of the bearing force in the event of a system failure. Take the second failure mode 
for example, assuming that the rotor is at the magnetic center, we obtain the following results:

$$
\begin{aligned}
& M_{1}=\left[\begin{array}{cc}
-1.18 & -0.59 \\
-0.59 & 0
\end{array}\right], \\
& M_{2}=\left[\begin{array}{ll}
80.19 & 0
\end{array}\right], \\
& M_{3}=\left[\begin{array}{cc}
0 & -0.59 \\
-0.59 & -1.18
\end{array}\right], \\
& M_{4}=\left[\begin{array}{ll}
0 & 80.19
\end{array}\right] .
\end{aligned}
$$

As seen from the above calculation results, elements of the coupling matrixes $M_{1}$ and $M_{3}$ are very small compared to the matrixes $M_{2}$ and $M_{4}$. Under normal circumstances, currents of the coil are approximately zero when the rotor is suspended in the center of the bias magnet flux. So we yield

$$
\begin{aligned}
& \left|I_{c}^{T} M_{1} I_{c}\right| \ll\left|M_{2} I_{c}\right|, \\
& \left|I_{c}^{T} M_{3} I_{c}\right| \ll\left|M_{4} I_{c}\right| .
\end{aligned}
$$

That means the first item of (13) can be neglected when the coil currents are small. Therefore, the forces and currents at the adjacent channels are decoupled at the magnetic center when the coil/amplifier fails. This is a distinct advantage of the four-pole PMRMB which differs from the traditional eightpole PMRMB.

Based on the analysis above, the approximate model of the bearing forces in the second failure mode can be written as

$$
\begin{aligned}
& f_{x}=80.19 i_{c x}+k_{h} h_{x}, \\
& f_{y}=80.19 i_{c y}+k_{h} h_{y} .
\end{aligned}
$$

Similarly, the force/current relations in the other failure mode are also approximate decoupling. Therefore, the force/ current relationship between the adjacent channels is approximate decoupling in any position of the protective gap after the failure of amplifier/coil without having to use the current distribution matrix to achieve the decoupling of the bearing force.

Therefore, taking into account the various failure modes and using the Taylor series expansion, the fault model of the bearing force can be described as

$$
\begin{aligned}
& f_{x}=\frac{1}{2}\left(k_{x 1}+k_{x 2}\right) k_{i} i_{c x}+k_{h} h_{x}, \\
& f_{y}=\frac{1}{2}\left(k_{y 1}+k_{y 2}\right) k_{i} i_{c y}+k_{h} h_{y},
\end{aligned}
$$

where $k_{x 1}, k_{x 2}, k_{y 1}$, and $k_{y 2}$ are elements of matrix $K ; k_{i}$ and $k_{h}$ are nominal current stiffness and nominal displacement stiffness.
3.3. Calculating of the Load Capacity. According to the gyro dynamic and gyro moment theory, the load capacity provided by the $x$ and $y$ channels of the radial MB are as follows:

$$
\begin{aligned}
& f_{a x}=\frac{H \omega_{g}}{2 l_{m}}, \\
& f_{b x}=-\frac{H \omega_{g}}{2 l_{m}}, \\
& f_{a y}=\frac{1}{2} m g \cos \left(\omega_{g} t+\varphi_{0}\right), \\
& f_{b y}=\frac{1}{2} m g \cos \left(\omega_{g} t+\varphi_{0}\right),
\end{aligned}
$$

where $m$ is the mass of the rotor, $a$ and $b$ are two poles of the rotor, $f_{x}$ and $f_{y}$ are the magnetic forces in the $x$-and $y$-direction, $g$ is the gravity acceleration, $H$ is the angular momentum of the rotor, $l_{m}$ refers to the distance between the central point of radial $\mathrm{MB}$, and rotor, $\omega_{g}$, is the gimbal angular rate.

If one of the amplifiers failed, then the bearing would be unable to provide sufficient magnetic force and the gyro moment would be reduced. Therefore, variation of the maximum load capacity before and after failure is also an important fault tolerance indicator of the MB. Maxwell's electromagnetic law applied to Figure 7 yields

$$
\begin{aligned}
& \phi_{x 1}=\phi_{\mathrm{pm} x 1}+\lambda_{x 1} i_{x 1}-\lambda_{x 2} i_{x 2}, \\
& \phi_{x 2}=\phi_{\mathrm{pm} x 2}+\lambda_{x 2} i_{x 2}-\lambda_{x 1} i_{x 1},
\end{aligned}
$$

where

$\phi_{\mathrm{pm} x 1}=\frac{H_{\mathrm{pm}} l_{\mathrm{pm}}}{R_{\mathrm{sum}}} \frac{R_{x 2}}{R_{x 1}+R_{x 2}}, \quad \phi_{\mathrm{pm} x 2}=\frac{H_{\mathrm{pm}} l_{\mathrm{pm}}}{R_{\mathrm{sum}}} \frac{R_{x 1}}{R_{x 1}+R_{x 2}}$,

$R_{\text {sum }}=R_{\mathrm{pm}}+\frac{R_{x 1} R_{x 2}}{R_{x 1}+R_{x 2}}+\frac{R_{y 1} R_{y 2}}{R_{y 1}+R_{y 2}}, \quad \lambda_{x 1}=\frac{N i_{x 1}}{R_{x 1}+R_{x 2}}$,

$\lambda_{x 2}=\frac{N i_{x 2}}{R_{x 1}+R_{x 2}}$

where $\phi_{\mathrm{pm} x 1}$ and $\phi_{\mathrm{pm} x 2}$ are bias flux density of the $x$ and $y$ channels. They are always set to equal to $B_{s} A / 2$ to obtain maximum magnetic forces at the point of magnetic material saturation.

The magnetic force of the $x$ channel can be given as

$$
f_{x}=\frac{\phi_{x 1}^{2}-\phi_{x 2}^{2}}{2 \mu_{0} A}
$$

The magnetic force along the $x$-direction becomes a maximum

$$
f_{x \max }=\frac{\phi_{x 1}^{2}-\phi_{x 2}^{2}}{2 \mu_{0} A}=\frac{B_{s}^{2} A}{2 \mu_{0}}
$$

when

$$
\lambda_{x 1} i_{x 1}=-\lambda_{x 2} i_{x 2}= \pm \frac{B_{s} A}{4} .
$$




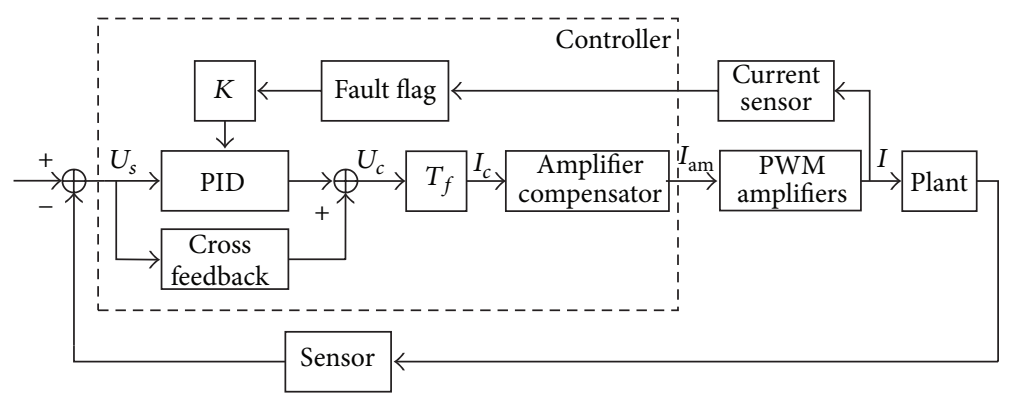

FIGURE 8: Diagram of the fault-tolerant control system.

Under normal circumstances

$$
f_{x \max }=\frac{B_{s}^{2} A}{2 \mu_{0}}>\frac{H \omega_{g}}{2 l_{m}} .
$$

Now we analyze the magnetic force of the $x$ channel when the amplifier is fault and on the assumptions that coil X2 is disconnected. Then the magnetic force of the $x$ channel after failure is given as

$$
f_{x}^{\prime}=\frac{\left(\phi_{\mathrm{pm} x 1}+\lambda_{x 1} i_{x 1}^{\prime}\right)^{2}-\left(\phi_{\mathrm{pm} x 2}-\lambda_{x 1} i_{x 1}^{\prime}\right)^{2}}{2 \mu_{0} A} .
$$

Because the currents of coil $\mathrm{X} 1$ and $\mathrm{X} 2$ are equal and opposite, let

$$
i_{x 1}^{\prime}=2 i_{x 1}
$$

and we can get the maximum magnetic force of the $x$ channel

$$
f_{x \max }^{\prime}=\frac{B_{s}^{2} A}{2 \mu_{0}}=f_{x \max } .
$$

Therefore, the maximum load capacity of four-pole PMRMB remains equivalent before and after failure. However, other types of multipole magnetic bearing usually do not have this feature. Thus the four-pole PMRMB is suitable for fault-tolerant control of SGMSCMG which has to output the rated torque at any conditions.

\section{Fault-Tolerant Control}

4.1. Design of the Fault-Tolerant Controller. Figure 8 shows the schematic diagram of the fault tolerant control system. The system mainly uses the decentralized PID and cross-feedback method to control the rotor at a high speed. If the amplifier/ coil failed, the current sensor would detect the fault signal and send it to the controller. Then the matrix $K_{a b}$ which includes fault flag of the eight channels of amplifier at two poles of the rotor will be updated in real time. Subsequently, controller will turn off the power amplifiers of these poles and implement the corresponding current distribution matrix $T_{f}$ for the remaining poles. In addition, variety of the coil currents will affect the nutation stability of the high-speed rotor and the low-pass filter is designed to compensate the bandwidth of the nonlinear PWM amplifier.
The output of controller $U_{c}$, input of amplifier $I_{c}$, and matrix $K_{a b}$ can be defined as

$$
\begin{aligned}
& U_{c}=\left[\begin{array}{llll}
u_{c a x} & u_{c b x} & u_{c a y} & u_{c b y}
\end{array}\right]^{T}, \\
& I_{c}=\left[\begin{array}{llll}
I_{c a x} & I_{c b x} & I_{c a y} & I_{c b y}
\end{array}\right]^{T}, \\
& K_{a b}=\operatorname{diag}\left(\begin{array}{llllllll}
k_{a x 1} & k_{a x 2} & k_{a y 1} & k_{a y 2} & k_{b x 1} & k_{b x 2} & k_{b y 1} & k_{b y 2}
\end{array}\right) \text {, }
\end{aligned}
$$

where $a$ and $b$ in $K_{a b}$ represent the two poles of the rotor.

According to (21), we know the current stiffness of the magnetic bearing will decrease after failure. In order to compensate the current stiffness after failure, let

$$
I_{c}=T_{f} U_{c}
$$

where

$$
\begin{gathered}
T_{f}=\operatorname{diag}\left[\frac{2}{k_{a x 1}+k_{a x 2}} \frac{2}{k_{a y 1}+k_{a y 2}} \frac{2}{k_{b x 1}+k_{b x 2}} \frac{2}{k_{b y 1}+k_{b y 2}}\right], \\
\left(k_{a x 1}+k_{a x 2} \neq 0, k_{a y 1}+k_{a y 2} \neq 0, k_{b x 1}+k_{b x 2} \neq 0, k_{b y 1}+k_{b y 2} \neq 0\right) .
\end{gathered}
$$

The matrix $T_{f}$ can be considered as redistribution to the currents of all channels. This ensures that the force/current gains are approximately unaltered before and after the failure. The meaning of the $T_{f}$ is that we only have to increase the currents of the normal coil to maintain the stability of the system when the other pole of this channel are failed. This method is easy to carry out and has a high reliability.

However, with the increased coil currents, there is a great increase of the noises introduced into the control structure which make the DSP noneffective to distinguish the nutation information included in the displacements of the high-speed rotor and also can lead the input signal to saturation and the system to instability. In a real system some practical limitations take place, because the gain values should not be too high in order to avoid saturation of the input of the system or even not to amplify the unavoidable noises and disturbances on the measurements that could affect the stability of the system. Therefore, it is necessary to adjust the parameters of the PID controller in order to obtain the desired tracking performances. 


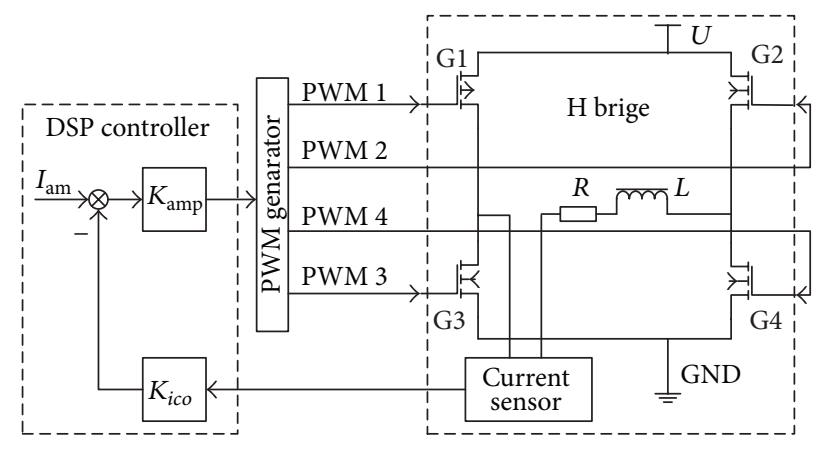

Figure 9: PWM Amplifier.

4.2. Compensate of the Amplifier. The key idea of fault tolerant control is currents redistribution. In addition, we have to consider the influence of the strong gyroscopic effects of the rotor at high rated speed. According to the analysis of the currents redistribution above, the currents of the normal coil are increased when the other pole of this channel failed. However, due to the nonlinear of the PWM amplifier, increasing of the coil currents will cause the phase lag of the amplifier which would seriously affect the nutation mode stability of the highspeed rotor.

The schematic of the PWM switching power amplifiers is shown in Figure 9. Where $R$ and $L$ are the equivalent resistance and inductance of the coil, $U$ is the DC power. The PWM amplifiers adopted $\mathrm{H}$-bridge structure has been widely used in magnetic bearing system. The coils of the magnetic bearing are inductive load. When using the amplifier to drive an inductive load, the alternating current in the coil reaches the limit of maximum slope and the limited bandwidth creates a stability problem [17]. The maximum slope can be expressed as

$$
\frac{d i}{d t}=\frac{U-R i}{L}
$$

If we define the input signal as

$$
i=i_{\max } \sin (\omega t),
$$

ignored the small resistance of the coil, the maximum slope must satisfy the following constraint condition

$$
\frac{d i_{\max }}{d t} \leq \frac{U}{\omega L}
$$

so that the amplifiers can completely track the input signal in real time and have no lag in phase.

Figure 10 shows the frequency characteristic of the amplifier with different amplitude of the input signal. According to the thick line and thin line showen in the figure, we can conclude that the phase lag in the high-frequency region is larger when the amplitude of the input signal is bigger. The phase lag of the amplifier can decrease the system stability and especially the nutation stability of the high-speed rotor. The nutation frequency is in direct proportionality to the rotor speed and can be approximately described as

$$
f_{\text {nat }}=\Omega \frac{J_{z}}{J_{r}}
$$

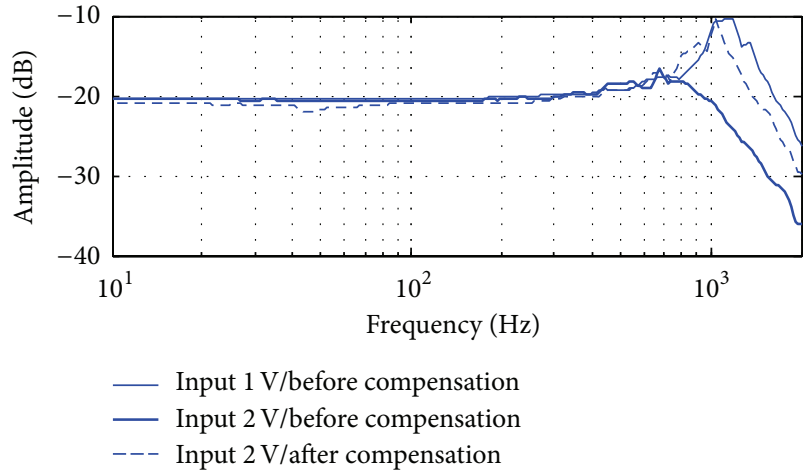

(a)

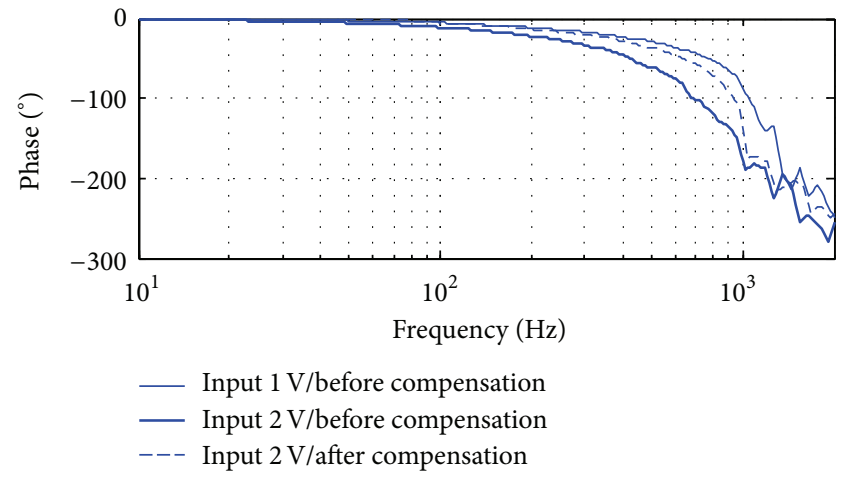

(b)

FIGURE 10: Frequency characteristic of the amplifier with different amplitude of the input signal.

where $f_{\text {nat }}$ is the nutation frequency of the rotor; $\Omega$ is the rotor speed; and $J_{r}$ and $J_{z}$ are the moments of inertia of the rotor about radial and axial direction.

For the SGMSCMG, its rated rotor speed is $20000 \mathrm{rpm}$, and its rated nutation frequency needed to be controlled is about $510 \mathrm{~Hz}$ (since $J_{z} / J_{r} \approx 1.55$ ). Therefore, we should care more about the frequency characteristic of the amplifier at the region around $510 \mathrm{~Hz}$ when we design a new controller. The phase of the amplifier can be increased by adding a compensated filter in the feedforward path [18]. A second-order filter is introduced into this subsystem, whose transfer function is given by

$$
G_{\mathrm{cmp}}(s)=\frac{3 s^{2}+8000 s+0.75 \times 10^{7}}{0.25 s^{2}+6200 s+1.72 \times 10^{7}} .
$$

The dashed line in Figure 10 shows the frequency characteristic of the amplifier after compensation. The comparison demonstrates that both the amplitude variation and the phase lag of the signal path are reduced by this filter.

\section{Experimental Setup and Results}

5.1. Experimental Setup. Figure 11 shows the picture of the experimental setup. For the amplifier fault-detection, halltype current sensors (LA-N25) are used to monitor the coil currents. The fault signal is flagged when absolute value of the actual current is zero or greater than $U / R L$ for the duration of 


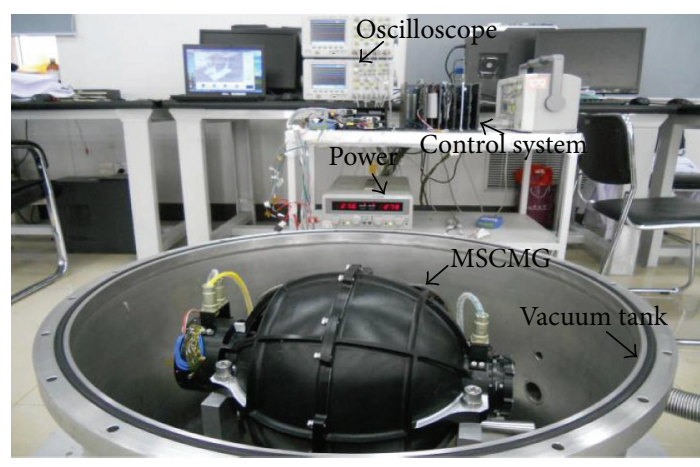

FIGURE 11: Experimental setup.

more than 3 sample period, in consideration of the dynamic bandwidth of the amplifiers and the accuracy of the current sensors. If the controller receives this fault signal, a new current distribution matrix is selected according to the fault condition. The PID controller, low-pass filter and high-pass filter proposed in the Figure 6 can be described as follows:

$$
\begin{aligned}
G_{\mathrm{PID}} & =K_{P}+\frac{K_{I}}{s}+\frac{K_{D}}{T_{D} s+1}, \\
G_{\mathrm{LPF}} & =\frac{1}{\left(T_{L} s\right)^{2}+2 \varepsilon_{L} T_{L} s+1}, \\
G_{\mathrm{HPF}} & =\frac{\left(T_{H} s\right)^{2}}{\left(T_{H} s\right)^{2}+2 \varepsilon_{H} T_{H} s+1},
\end{aligned}
$$

where $T_{H}=1 / 1.8 \Omega$.

All the parameters of the SGMSCMG used in the experiments are given in the Table 3. The proposed control algorithm is implemented in a TMS320C32 digital-signal-processor-based control computer with the sampling frequency of $6.67 \mathrm{kHz}$

5.2. Fault-Tolerant Capacity and Robust Performance. The experiment is carried out to illustrate operation and reliability of the fault tolerant magnetic bearing system under the condition that $\Omega=20000 \mathrm{rpm}, \omega_{g}=10^{\circ} / \mathrm{s}$, and $\varphi_{0}=0^{\circ}$. The amplitude of the rotor displacements is about $15 \mu \mathrm{m}$ on account of mass unbalance. Take the first failure mode for example. Figure 12 shows the rotor motion when the coil AX2 in the four-pole radial bearing is manually disconnected from the amplifiers at $0.5 \mathrm{~s}$. With transient regulation after failure, the rotor is still suspending nearby the magnetic center and the peak-to-peak amplitude of the displacement is almost changeless. This indirectly demonstrate that the load capacity also satisfy the requirement of CMG to output the rated torque.

The coil currents after failure are shown in Figure 13. The peak-to-peak current amplitude of the coil AX1 is about $200 \mathrm{~mA}$ before failure and $400 \mathrm{~mA}$ after failure. Therefore, the phase lag of the amplifier will increase with the change rate of the coil current and the stability of the system will be destroyed if the PWM amplifier has not been compensated.
TABLE 3: SGMSCMG specifications.

\begin{tabular}{lc}
\hline Parameter & Value \\
\hline$m(\mathrm{~kg})$ & 3.2 \\
$J_{z}\left(\mathrm{~kg} \cdot \mathrm{m}^{2}\right)$ & 0.006569 \\
$J_{x}\left(\mathrm{~kg} \cdot \mathrm{m}^{2}\right)$ & 0.004236 \\
$K_{i}(\mathrm{~N} / \mathrm{A})$ & 160 \\
$K_{h}(\mathrm{~N} / \mu \mathrm{m})$ & -0.57 \\
$U(\mathrm{~V})$ & 28 \\
$L(\mathrm{mH})$ & 12 \\
$K_{P}$ & 0.32 \\
$K_{I}$ & 4.5 \\
$K_{D}$ & 0.00065 \\
$T_{D}$ & 0.0002 \\
$K_{x l}$ & 0.30 \\
$K_{x h}$ & 1.20 \\
$K_{c}$ & 0.90 \\
$T_{L}$ & 0.0019 \\
$\varepsilon_{L}$ & 0.75 \\
$\varepsilon_{H}$ & 0.80 \\
\hline
\end{tabular}

Figure 14 demonstrates the rotor displacements when the coils AX1 and AX2 are disconnected successively at $0.3 \mathrm{~s}$ and $0.7 \mathrm{~s}$. The corresponding currents in every channel are shown in Figure 15. Even with two poles failing, the suspension is maintained after a slight adjustment of the center position even though the gimbal is rotating at $10 \%$.

In addition, the nutation amplitude is so small when the rotor is stable that we cannot observe directly from the rotor displacements. In order to compare the change of the rotor nutation expediently before and after failure, the FFT is introduced in the system. Figure 16 shows the spectrums obtained by Agilent Oscilloscope (DXO-X 3014A) which implement the fast Fourier transform algorithm to the original rotor displacements when the rotor speed is $333 \mathrm{~Hz}$. However, there are much high-frequency noises in the system, the nutation frequency that we see from the oscilloscope after FFT is not only one but also a tuft. We can see from the figure that the nutation frequency is about $530 \mathrm{~Hz}$ (since $J_{z} / J_{r} \approx 1.55$ ) and the nutation amplitude changes when the coil is disconnected at the same rotor speed. The most intuitionistic and simplest method to estimate whether the rotor is stable when the rotor is at a high speed is the size of the nutation amplitude. Increasing of the nutation amplitude indicates that the stabilization margin gets smaller and the motion of the nutation becomes unstable gradually.

The upper and middle spectrums are obtained before and after the coils AX1 and AX2 were disconnected. The maximum nutation amplitude after failure is $-43 \mathrm{~dB}$ which is much higher compared to the upper spectrum. That indicates the nutation stability is affected by the failure. As a matter of fact, it was mainly aroused by the increasing of the coil currents which have been analyzed in Section 4 . Therefore, we have to design a compensated filter to increase the bandwidth of the amplifiers. The third picture shows the nutation amplitude spectrum after being compensated. The amplitude is 


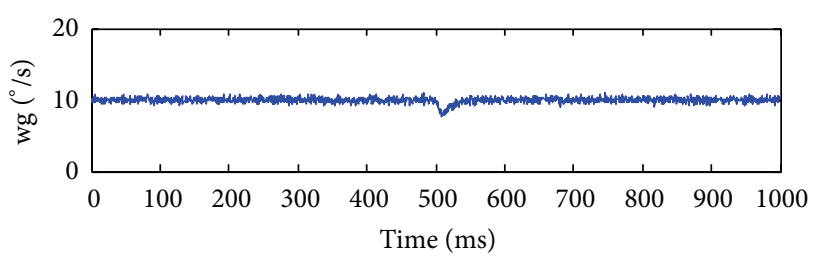

(a)

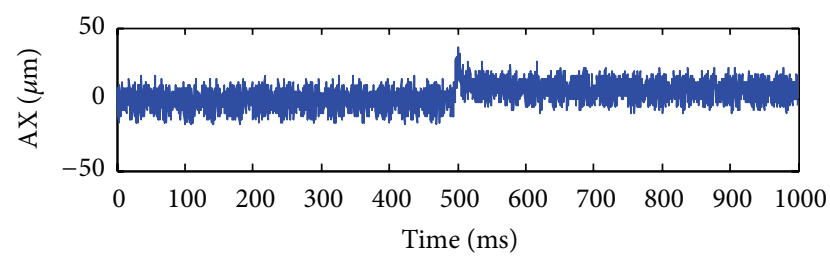

(b)

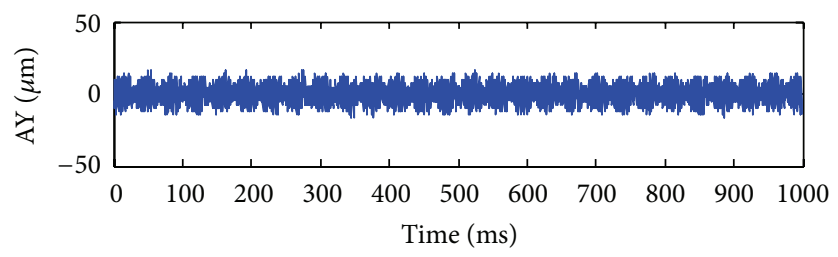

(c)

FIgURE 12: Rotor displacements when coil AX2 is failing.

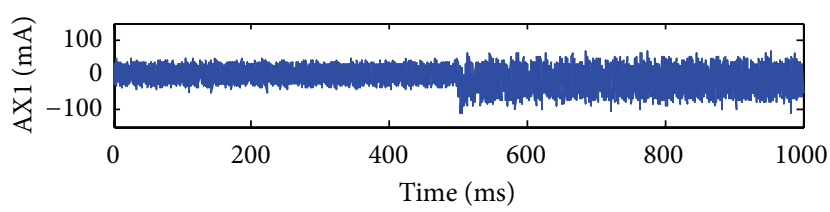

(a)

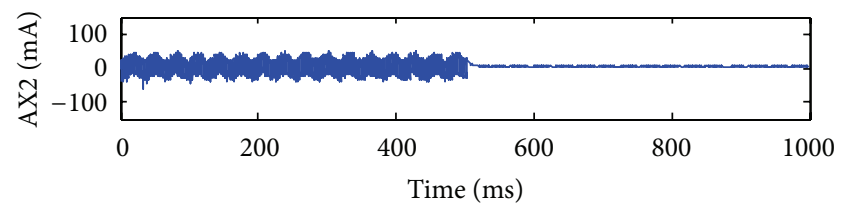

(c)

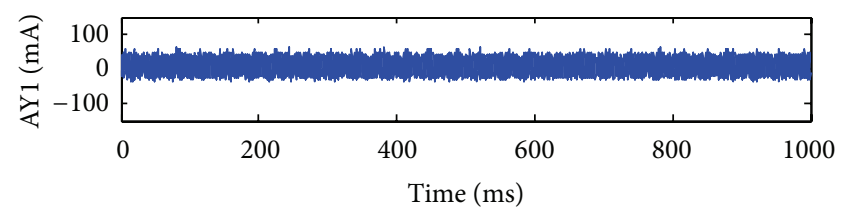

(b)

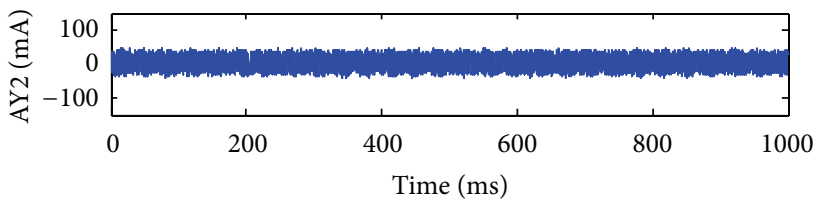

(d)

FIgURE 13: Coil currents when coil AX2 is failing.

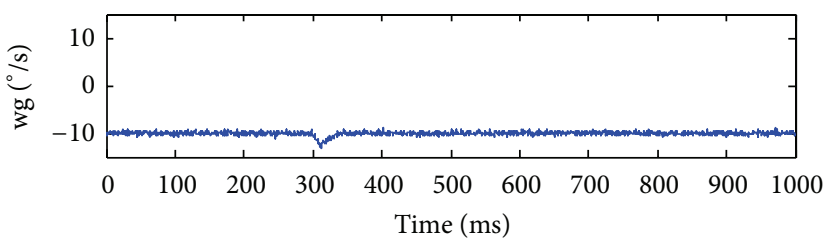

(a)

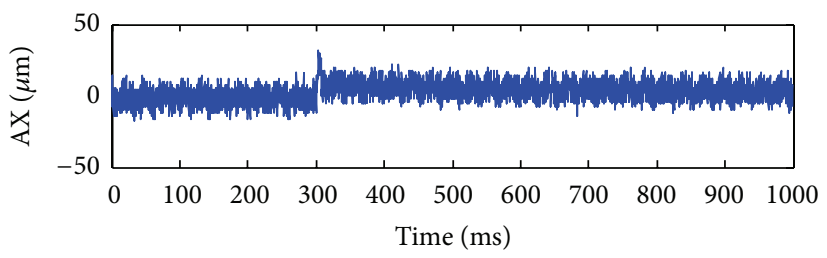

(b)

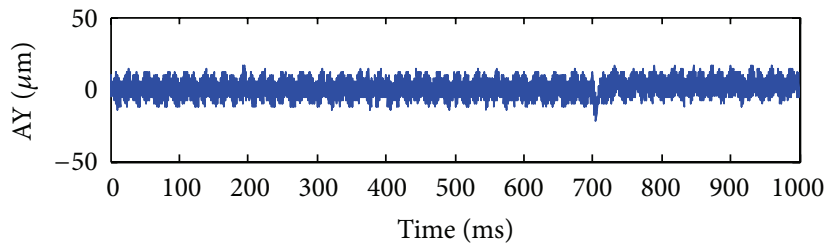

(c)

FIgURE 14: Rotor displacements when coils AX2 and AY2 are failing. 


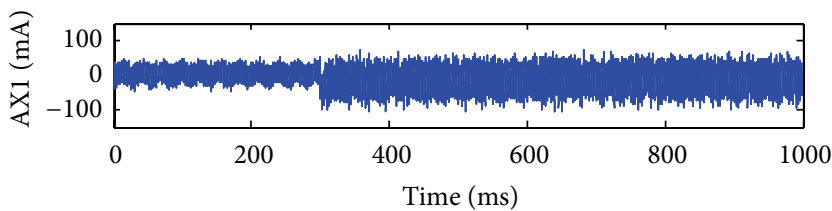

(a)

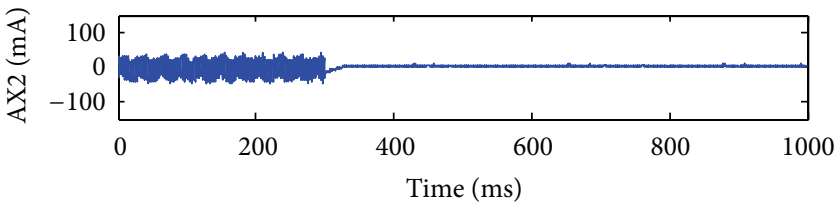

(c)

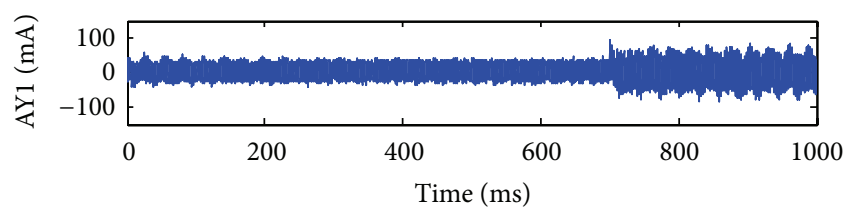

(b)

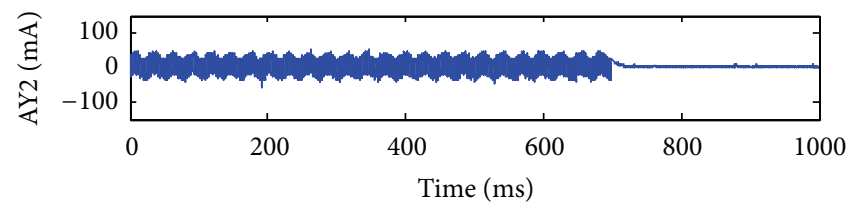

(d)

FIGURE 15: Coil currents when coils AX2 and AY2 are failing.

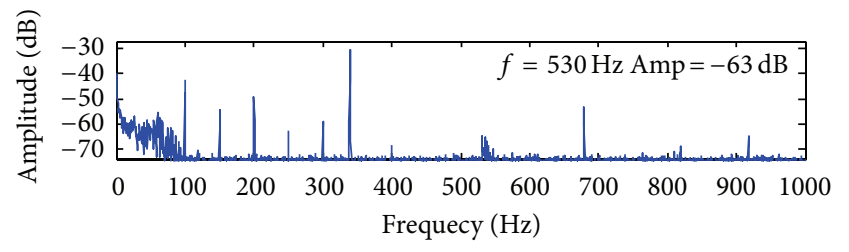

(a)

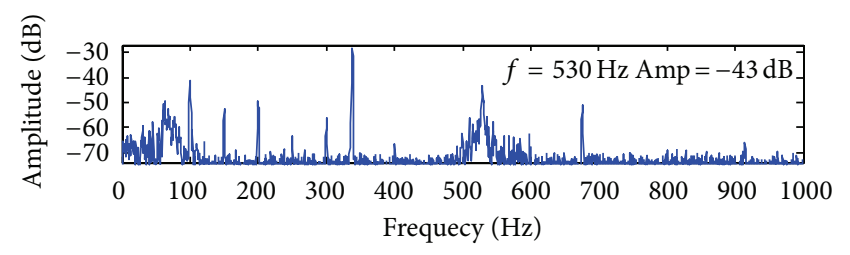

(b)

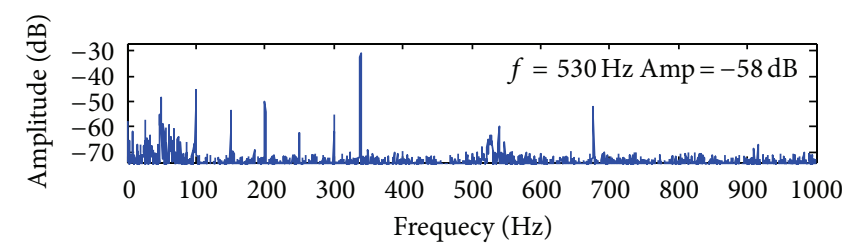

(c)

FIGURE 16: Spectrums of the FFT for rotor displacements. (a) Spectrum before failure. (b) Spectrum after failure. (c) Spectrum after compensated.

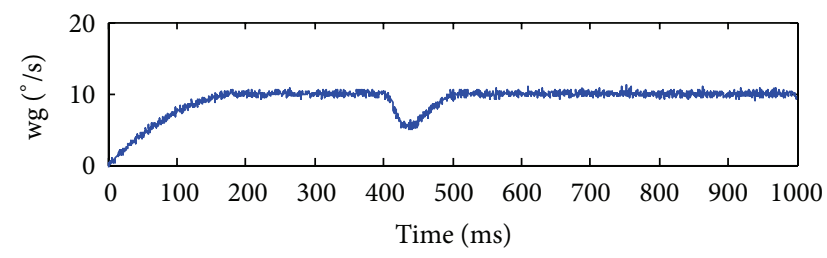

(a)

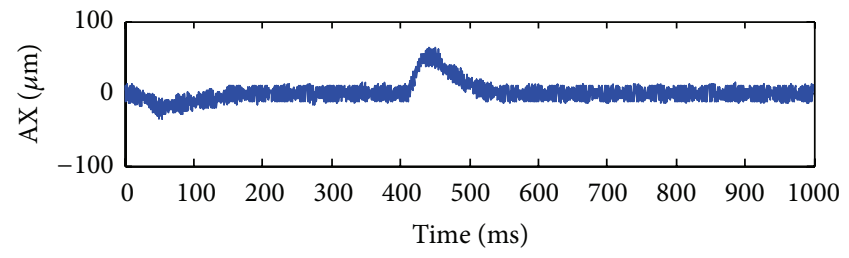

(b)

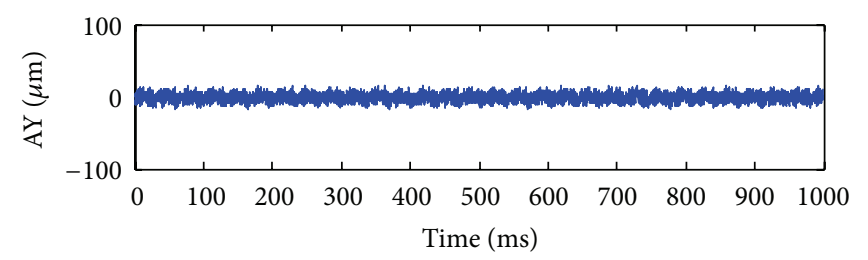

(c)

FIGURE 17: The rotor displacements response after failure with external disturbance on the gimbal. 
decreased to $-58 \mathrm{~dB}$ and is almost the same to the amplitude before failure.

In order to further verify the control performances of disturbance rejection especially the moving-gimbal effects of the proposed fault-tolerant control system after failure, reference angular rate step, and external disturbance are imposed on the system, respectively. The experiment is carried out under the rated rotor speed when the coils AX2 and AY2 are disconnected. Firstly, the reference gimbal angular rate steps from 0 to $10^{\circ} / \mathrm{s}$ at $0 \mathrm{~s}$. Secondly, an intense external disturbance is imposed on the gimbal at $0.4 \mathrm{~s}$. The rotor displacements response is shown in Figure 17. We see that the rotor is suspended well when the gimbal rate is stepping. Moreover, with the intense external disturbance on the gimbal, the rotor can recentralize quickly after acutely fluctuating in which the maximum amplitude has reached $60 \%$ of the air gap. These all demonstrate that the proposed fault-tolerant control system has good disturbance rejection and strong robust performance and meet for the requirements of the SGMSCMG.

\section{Conclusions}

In this paper, we presented a fault tolerant magnetic bearing system for SGMSCMG. In view of the indispensable performance for low steady-state power losses, the four-pole PMRMB is designed so that the rotating loss can be reduced as little as we can. Furthermore, considering the complicated dynamical characteristic of the SGMSCMG especially the movinggimbal effects and gyroscopic effects, the structure of the magnetic bearing introduced in this paper is different from the conventional. The current distribution matrix and compensation filters for amplifiers are designed to realize a stable suspending of the high-speed rotor after failing. The experimental results demonstrate the adequacy of the fault-tolerant magnetic bearing system.

\section{Conflict of Interests}

The authors declare that there is no conflict of interests regarding the publication of this paper.

\section{Acknowledgment}

This work is supported by the Aviation Science Fund of China under Grant 2012ZB51019 and in part by the Cultivation and Development Project of Science and Technology Innovation Base of Beijing under Grant Z131104002813105.

\section{References}

[1] W. E. Haynes, "Control moment gyros for the space shuttle," in Proceedings of the IEEE Position Location and Navigation Symposium, pp. 113-115, New York, NY, USA, 1984.

[2] S. Zheng and B. Han, "Investigations of an integrated angular velocity measurement and attitude control system for spacecraft using magnetically suspended double-gimbal CMGs," Advances in Space Research, vol. 51, no. 12, pp. 2216-2228, 2013.
[3] E. H. Maslen and D. C. Meeker, "Fault tolerance of magnetic bearings by generalized bias current linearization," IEEE Transactions on Magnetics, vol. 31, no. 3, pp. 2304-2314, 1995.

[4] D. C. Meeker, Optimal solutions to the inverse problem in quadratic magnetic actuators [Ph.D. dissertation], University of Virginia, 1996.

[5] E. H. Maslen, C. K. Sortore, G. T. Gillies, R. D. Williams, S. J. Fedigan, and R. J. Aimone, "Fault tolerant magnetic bearings," Journal of Engineering for Gas Turbines and Power, vol. 121, no. 3, pp. 504-508, 1999.

[6] U. J. Na and A. Palazzolo, "Optimized realization of fault-tolerant heteropolar magnetic bearings," Journal of Vibration and Acoustics, vol. 122, no. 3, pp. 209-221, 2000.

[7] U. J. Na, A. B. Palazzolo, and A. Provenza, "Test and theory correlation study for a flexible rotor on fault-tolerant magnetic bearings," Journal of Vibration and Acoustics, vol. 124, no. 3, pp. 359-366, 2002.

[8] U. J. Na and A. B. Palazzolo, "Fault tolerance of magnetic bearings with material path reluctances and fringing factors," IEEE Transactions on Magnetics, vol. 36, no. 6, pp. 3939-3946, 2000.

[9] M. D. Noh, S.-R. Cho, J.-H. Kyung, S.-K. Ro, and J.-K. Park, "Design and implementation of a fault-tolerant magnetic bearing system for turbo-molecular vacuum pump," IEEE/ASME Transactions on Mechatronics, vol. 10, no. 6, pp. 626-631, 2005.

[10] M. Ahrens, L. Kučera, and R. Larsonneur, "Performance of a magnetically suspended flywheel energy storage device," IEEE Transactions on Control Systems Technology, vol. 4, no. 5, pp. 494-502, 1996.

[11] M.-H. Li, A. B. Palazzolo, A. Kenny, A. J. Provenza, R. F. Beach, and A. F. Kascak, "Fault-tolerant homopolar magnetic bearings," IEEE Transactions on Magnetics, vol. 40, no. 5, pp. 33083318, 2004.

[12] T. Wei and J.-C. Fang, "Moving-gimbal effects and angular rate feedforward control in magnetically suspended rotor system of CMG," Journal of Astronautics, vol. 26, no. 1, pp. 19-23, 2005 (Chinese).

[13] J. Fang and Y. Ren, "High-precision control for a single-gimbal magnetically suspended control moment gyro based on inverse system method," IEEE Transactions on Industrial Electronics, vol. 58, no. 9, pp. 4331-4342, 2011.

[14] J. Fang, S. Zheng, and B. Han, "AMB vibration control for structural resonance of double-gimbal control moment gyro with high-speed magnetically suspended rotor," IEEE/ASME Transactions on Mechatronics, vol. 18, no. 1, pp. 32-43, 2011.

[15] Y. Xu, Y. Dun, X. Wang, and Y. Kong, "Analysis of hybrid magnetic bearing with a permanent magnet in the rotor by FEM," IEEE Transactions on Magnetics, vol. 42, no. 4, pp. 1363-1366, 2006.

[16] Y. Okada, B. Nagai, and T. Shimane, "Cross feedback stabilization of the digitally controlled magnetic bearing," in Proceedings of the ASME Conference on Mechanical Vibration and Noise, Montreal, Canada, 1989.

[17] G. Schweitzer and E. H. Maslen, Magnetic Bearings, Theory, Design, and Application to Rotating Machinery, Springer, Berlin, Germany, 2009.

[18] M. Chen and C. R. Knospe, "Feedback linearization of active magnetic bearings: current-mode implementation," IEEE/ ASME Transactions on Mechatronics, vol. 10, no. 6, pp. 632-639, 2005. 


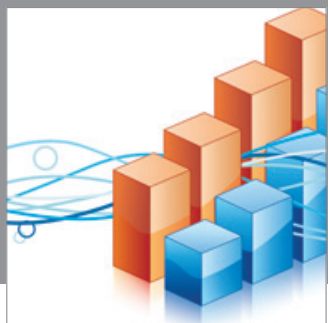

Advances in

Operations Research

mansans

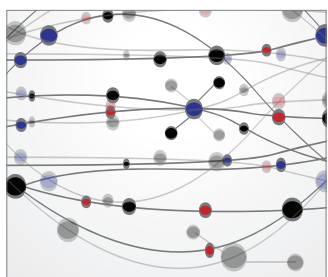

The Scientific World Journal
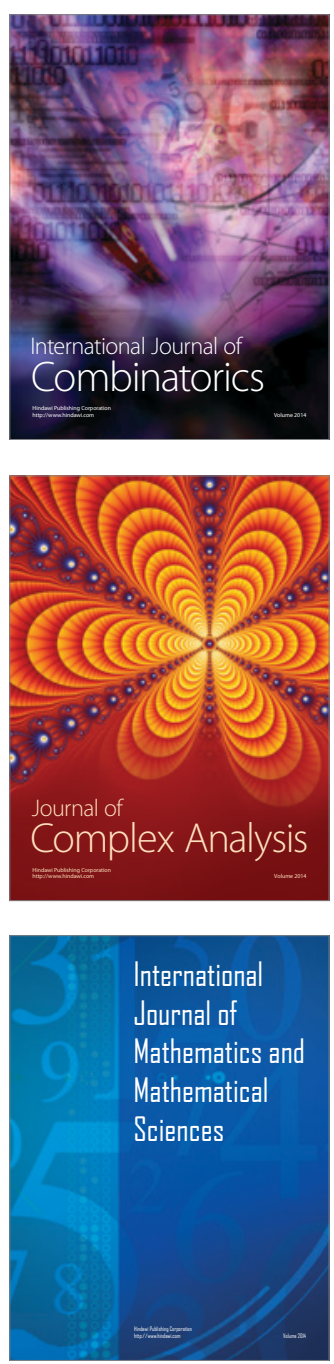
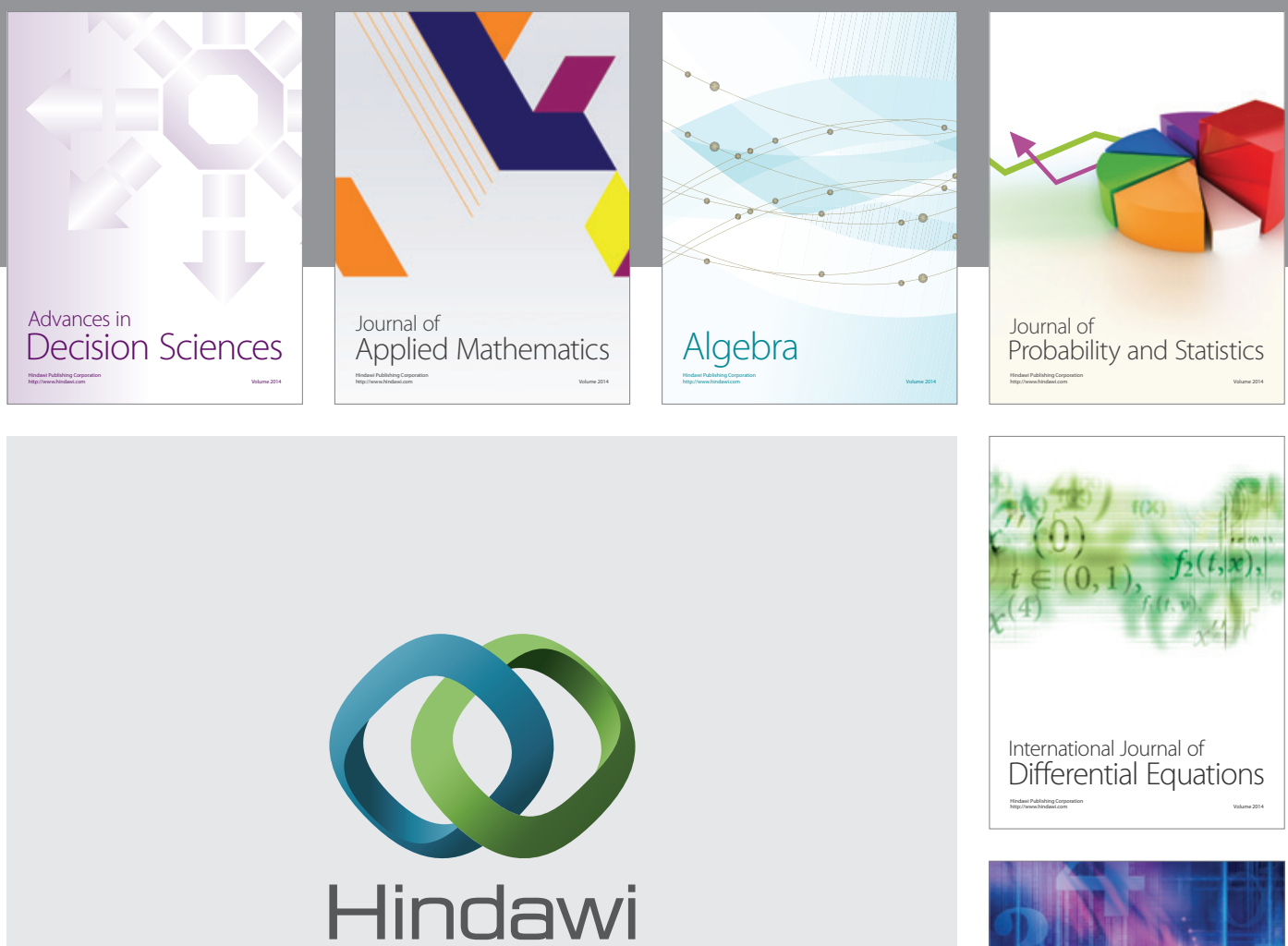

Submit your manuscripts at http://www.hindawi.com
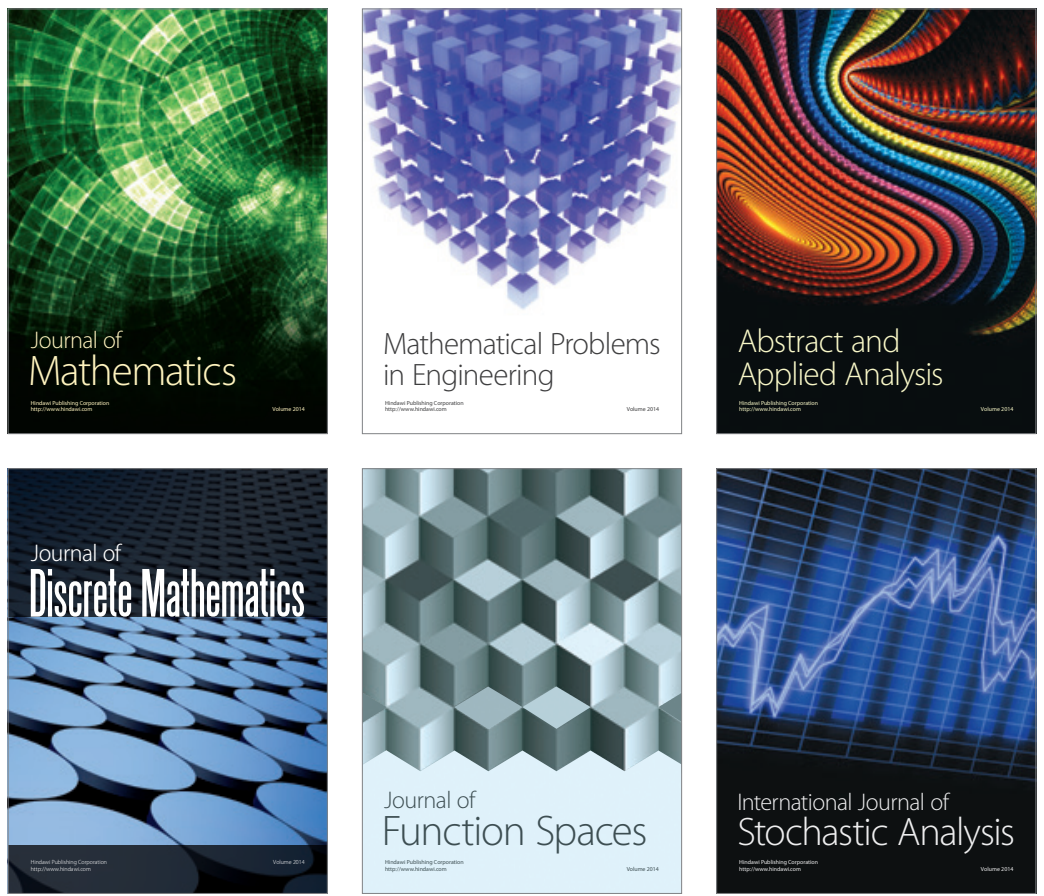

Journal of

Function Spaces

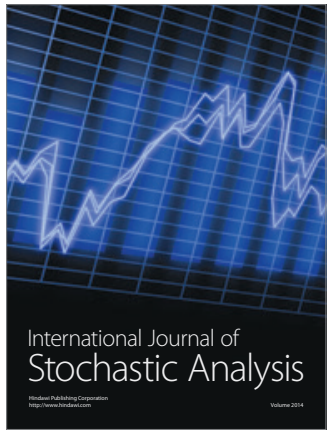

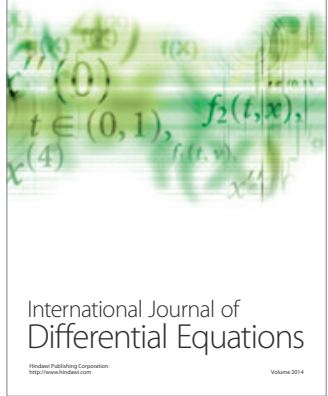
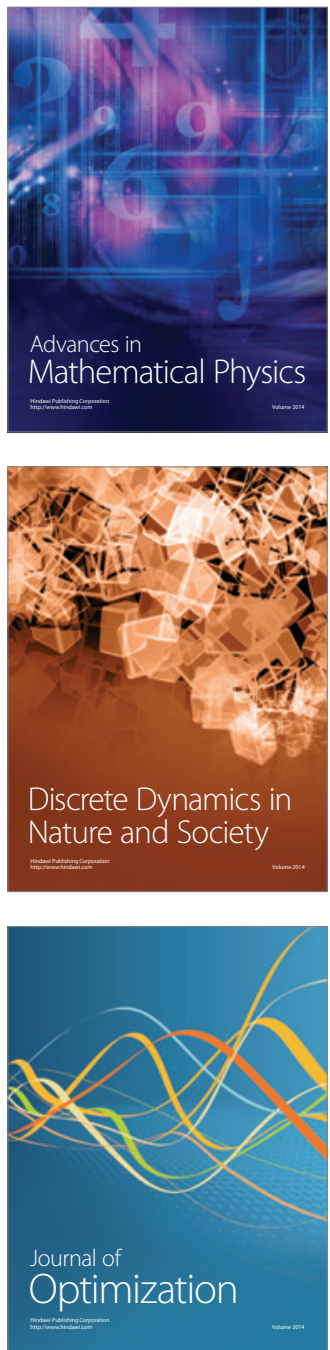